\title{
Publicly Accessible Rehabilitation Protocols for Acromioclavicular Joint Reconstruction Are Widely Variable
}

\author{
Sana G. Cheema, B.A., Christina Hermanns, B.S., Reed G. Coda, B.S., \\ Armin Tarakemeh, B.S., Scott M. Mullen, M.D., John Paul Schroeppel, M.D., \\ Bryan G. Vopat, M.D., and Mary K. Mulcahey, M.D.
}

\begin{abstract}
Purpose: The purpose of this study was to assess the availability and variability of publicly accessible acromioclavicular (AC) joint reconstruction rehabilitation protocols. Methods: Protocols were identified by searching the websites of orthopedic surgery residency programs in the United States located from the Fellowship and Residency Electronic Interactive Database Access System. Private practice groups with publicly available protocols were also included. Results: Twenty-one protocols were included for review. Four of $14(29 \%)$ protocols suggested starting passive range of motion (ROM) at postoperative week 2. Six of $20(30 \%)$ protocols recommended initiation of full ROM at 6 weeks. Active ROM beginning at 6 weeks was recommended by 6 of 20 (30\%) protocols. Six of 16 (38\%) protocols recommended initiating active assisted ROM at 6 weeks. Sling immobilization for 6 weeks was recommended by 8 of 18 ( $44 \%$ ) protocols. Shoulder isometric exercise initiated at 4 weeks was recommended by 4 of 13 (31\%) protocols. Seven of 21 $(33 \%)$ protocols recommended initiating shoulder strengthening at 12 weeks postoperatively. Return to sport time was included in $17(81 \%)$ protocols with a range of 12 to 48 weeks (mean, 22 weeks). Conclusions: There was substantial variability in publicly accessible AC joint rehabilitation protocols, including a wide range in the recommendations for appropriate time to return to sport. Although strengthening exercises, active ROM, and active assisted ROM were recommended by most protocols, there were considerable differences in recommendations for when to initiate these rehabilitation components. Clinical Relevance: Rehabilitation is important for outcomes of AC joint reconstruction. This study shows the variability present in rehabilitation recommendations among online-accessible AC joint reconstruction rehabilitation protocols.
\end{abstract}

$\mathbf{I}_{n}^{\mathrm{n}}$ njuries to the acromioclavicular (AC) joint represent nearly $40 \%$ to $50 \%$ of all athletic shoulder injuries. ${ }^{1}$ A study by Kaplan et al. ${ }^{2}$ found that of 336 elite collegiate American football players, 50\% had shoulder

From the Department of Orthopaedic Surgery, University of Kansas Health System, Kansas City, Kansas, U.S.A. (S.G.C., C.H., R.G.C., A.T., S.M.M., J.P.S., B.G.V.) and Department of Orthopaedic Surgery, Tulane University School of Medicine, New Orleans, Louisiana, U.S.A. (M.K.M.).

The authors report that they have no conflicts of interest in the authorship and publication of this article. Full ICMJE author disclosure forms are available for this article online, as supplementary material.

This article was presented as a poster presentation at the Orthopaedic Research Society Feb 8, 2020 Meeting; Phoenix, Arizona.

Received April 7, 2020; accepted October 12, 2020.

Address correspondence to Mary K. Mulcahey M.D., 1430 Tulane Ave, Box 8632,New Orleans, LA 70112,U.S.A.E-mail:mary.mulcahey.md@gmail.com

(C) 2020 THE AUTHORS. Published by Elsevier Inc. on behalf of the Arthroscopy Association of North America. This is an open access article under the CC BY-NC-ND license (http://creativecommons.org/licenses/by-nc-nd/4.0/).

2666-061X/20540

https://doi.org/10.1016/j.asmr.2020.10.007 injuries, with the most common specific injury to the shoulder being AC joint separation (40\%). Either direct or indirect mechanisms can lead to AC joint injury. Direct injury occurs as a result of a force applied to the acromion with the shoulder adducted, which usually results from a fall onto the superolateral aspect of the shoulder. ${ }^{1}$ Indirect injuries occur by a fall on an outstretched arm or elbow with a superiorly directed force. ${ }^{1}$ The Rockwood classification system is most commonly used to describe AC joint injuries. ${ }^{3}$ Treatment for type I to II injuries is usually nonoperative, whereas type IV to VI injuries frequently require surgical intervention. ${ }^{4}$

Rehabilitation following AC joint reconstruction plays an important role in recovery and return to play. ${ }^{5}$ There is currently no well-defined standard for postoperative rehabilitation for patients undergoing AC joint reconstruction, which may lead to variability in the rehabilitation practices used by physicians. Differences in postoperative rehabilitation protocols may also be 


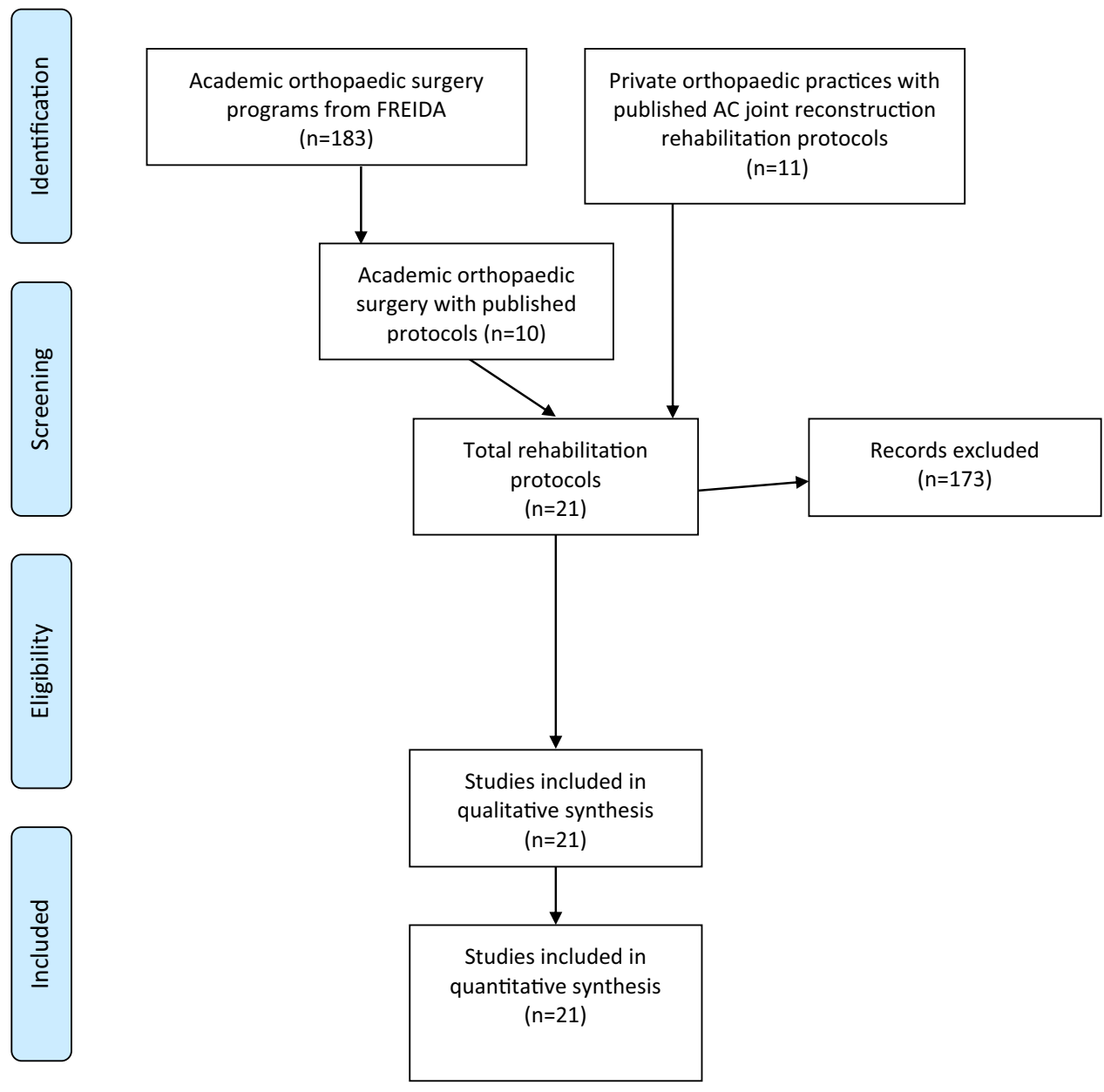

Fig 1. Flow diagram used for data collection in this study. (FREIDA, Fellowship and Residency Electronic Interactive Database Access System) influenced by the fixation technique used for AC joint reconstruction. According to Simovitch et al., ${ }^{1}$ surgical techniques for the treatment of AC joint dislocation can be categorized into 3 main groups: primary AC joint fixation, fixation between the coracoid process and clavicle using coracoclavicular screw and suture loop, and ligament reconstruction using autogenous hamstring, anterior tibialis allograft, or coracoacromial ligament transfer. Although open and arthroscopic AC joint reconstruction have no difference in outcomes

Table 1. Comparison of Protocols Based on Different Rehabilitation Components of AC Joint Reconstruction

\begin{tabular}{lcc}
\hline \multicolumn{1}{c}{ Rehabilitation Component } & $\begin{array}{c}\text { Total number } \\
\text { of protocols }\end{array}$ & $\begin{array}{c}\text { Percent } \\
\text { of protocols }\end{array}$ \\
\hline Passive Range of Motion & 14 & $67 \%$ \\
Full Range of Motion & 20 & $95 \%$ \\
Active Range of Motion & 20 & $95 \%$ \\
Active Assistive Range of Motion & 16 & $76 \%$ \\
Sling Immobilization & 18 & $86 \%$ \\
Isometric Shoulder Exercises & 13 & $62 \%$ \\
Shoulder Strengthening & 21 & $100 \%$ \\
Return to Sport & 17 & $81 \%$ \\
\hline
\end{tabular}

and complication rates, there can be a difference in rehabilitation time between the 2 surgical techniques. ${ }^{6}$

Previous studies that looked at online rehabilitation protocols for other orthopedic surgeries found a low number of accessible protocols and great variation in rehabilitation practices among academic orthopedic programs. ${ }^{7,8}$ The purpose of this study was to assess the

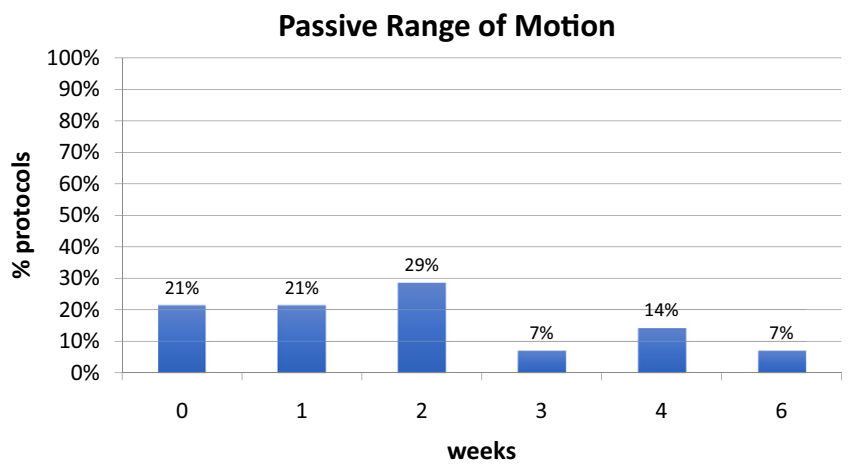

Fig 2. Recommendation by protocols for initiation of passive range of motion (in weeks) following acromioclavicular joint reconstruction. 


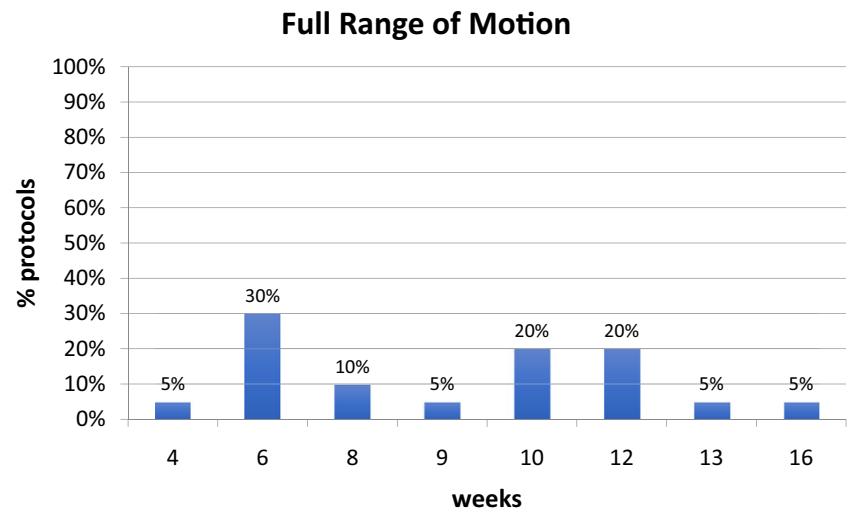

Fig 3. Recommendation by protocols for initiation of full range of motion (in weeks) following acromioclavicular joint reconstruction.

availability and variability of publicly accessible AC joint reconstruction rehabilitation protocols. We hypothesized that there would be a limited number of rehabilitation protocols available online and that there would be variability between the protocols with regard to initiation of certain exercises and timing of return to sport.

\section{Methods}

Publicly accessible AC joint rehabilitation protocols were collected from websites for academic orthopedic surgery programs and private orthopedic practice groups in the United States. Academic orthopedic residency programs were identified using the American Medical Association Fellowship and Residency Electronic Interactive Database (FREIDA). In May 2019, a search was performed on Google using the following search terms: the name of the orthopedic residency program and "AC joint reconstruction rehabilitation protocol." The search results were limited to the first page. In addition, US orthopedic private practice rehabilitation protocols were identified by searching Google
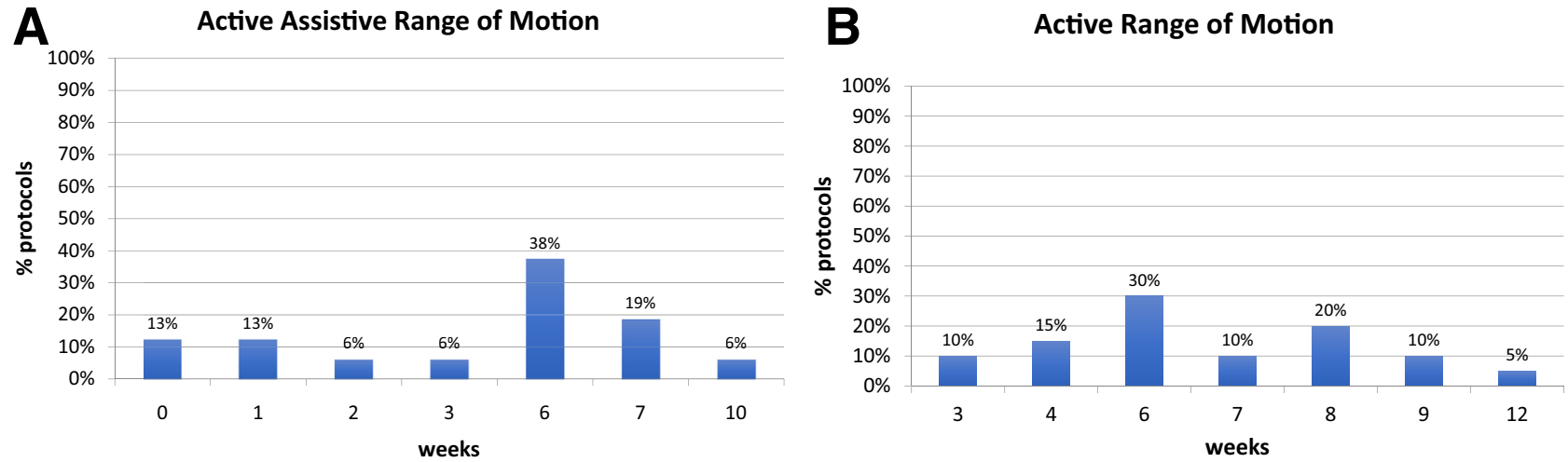

Fig 4. (A) Recommendation by protocols for initiation of active assistive range of motion (in weeks) following acromioclavicular (AC) joint reconstruction. (B) Recommendation for initiating active range of motion (in weeks) following AC joint reconstruction. 


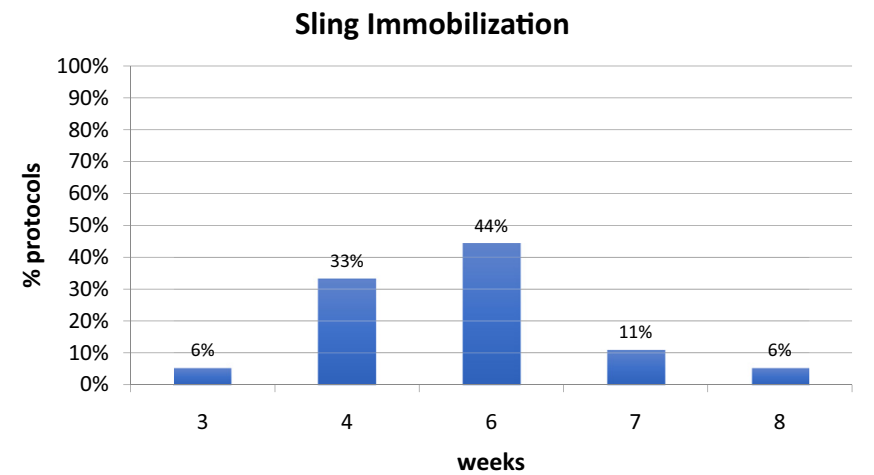

Fig 5. Recommendation by protocols for sling immobilization (in weeks) following acromioclavicular joint reconstruction.

\section{Range of Motion}

Shoulder range of motion can be subdivided into full, passive, active assisted, and active. If the protocol specified a range, the initial value of each range was used (e.g., if a protocol specified return to sport at 12-14 weeks, 12 weeks was used). The time to start PROM was included in $14(67 \%)$ protocols (Fig 2). The recommendation for range of time for beginning PROM was from 0 to 6 weeks. The greatest number of protocols recommended beginning PROM at 2 weeks ( 4 of $14[29 \%])$ following AC joint reconstruction.

The time to start FROM was included in 20 (95\%) protocols (Fig 3). The recommendation for range of time for beginning FROM was from 4 to 16 weeks. Six of $20(30 \%)$ protocols recommended starting FROM at 6 weeks.

Timing to initiate AAROM was included in $16(76 \%)$ protocols, and AROM was included in $20(95 \%)$ protocols (Fig $4 \mathrm{~A}$ and $\mathrm{B}$ ). The recommendation for range of time for initiating AAROM was from 0 to 10 weeks. Six of $16(38 \%)$ protocols recommended starting AAROM at 6 weeks. Recommendations for starting AROM ranged from 3 to 12 weeks. Six of $20(30 \%)$ protocols recommended starting AROM at 6 weeks.

\section{Sling}

The use of a sling was recommended by 18 (86\%) protocols. Recommendations for duration of sling immobilization ranged from 3 to 8 weeks (Fig 5). Eight of $18(44 \%)$ protocols recommended sling immobilization for 6 weeks. Some of the protocols did not provide a specific guideline for sling use within the rehabilitation protocol.

\section{Deltoid Isometric Exercises}

Deltoid isometric exercises of the shoulder were recommended by $13(62 \%)$ of the rehabilitation protocols. The time when isometric exercises should be performed in each protocol is demonstrated in Fig 6. Initiation of isometric exercises ranged from 0 to 6 weeks postoperatively. Starting these exercises at 4 weeks postsurgery was recommended by 4 of $13(31 \%)$ of the protocols.

\section{Strengthening}

All 21 protocols $(100 \%)$ recommended incorporating shoulder strengthening exercises. The time when strengthening should be performed according to each protocol is demonstrated in Fig 7. Initiation of strengthening ranged from 4 to 14 weeks. Seven of 21 $(33 \%)$ protocols recommended starting strengthening at 12 weeks postoperatively.

\section{Return to Sport}

Of the 21 protocols, $17(81 \%)$ included a recommendation for time to return to sport (RTS) (Fig 8). The recommended time to RTS ranged from 12 to 48 weeks. Seven of 17 protocols $(41 \%)$ recommended RTS at 16 weeks.

\section{Discussion}

This study found that only a small percentage of academic orthopedic surgery programs publish an online AC joint reconstruction rehabilitation protocol. The study also found tremendous variability between online published AC joint reconstruction rehabilitation protocols. Specifically, there was disagreement among the protocols on the best time to initiate most rehabilitation components. In addition, numerous protocols failed to include certain rehabilitation components altogether. For example, 7 of $21(33 \%)$ protocols did not include any recommendation on when to initiate PROM even though many initial goals to restore mobility after surgery are achieved using PROM exercises. ${ }^{5}$ In addition, a total of 11 rehabilitation protocols were identified out of the 183 academic orthopedic surgery residency programs evaluated in this study. Therefore, our hypothesis that there would be a limited number of rehabilitation protocols available online and that there

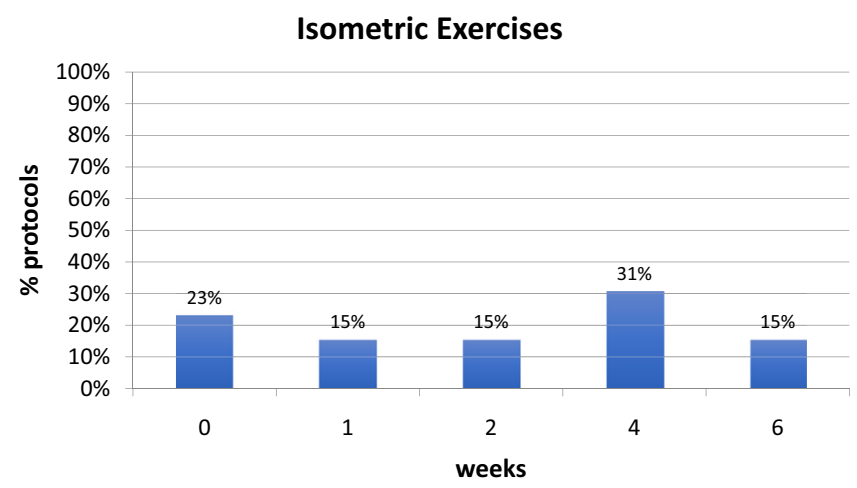

Fig 6. Recommendation by protocols for initiation of isometric exercises (in weeks) following acromioclavicular joint reconstruction. 


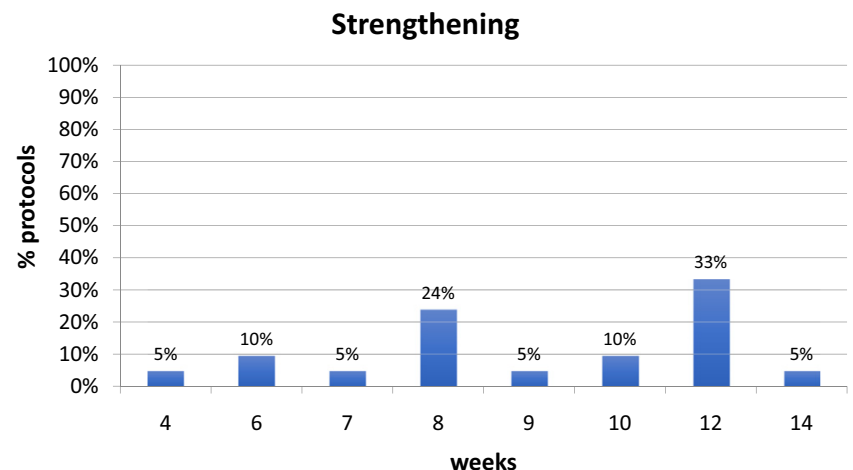

Fig 7. Recommendation by protocols for initiation of strengthening exercises (in weeks) following acromioclavicular joint reconstruction.

would be variability in recommendations made by the protocols was supported by the findings in this study.

All 21 protocols recommended including shoulder strengthening exercises as part of AC joint reconstruction rehabilitation; however, there was disagreement on when these strengthening exercises should begin. Five of 21 protocols $(24 \%)$ agreed that strengthening should begin at 8 weeks, and 7 of $21(33 \%)$ protocols agreed that strengthening should begin at 12 weeks. There was also considerable variation between protocols on when FROM exercises should be initiated. This could potentially be caused by FROM being defined differently by each program. For example, one program could mean full AROM while another could mean full AAROM when they both make recommendations for FROM within their protocols. There was also substantial variability in the protocols with regard to RTS following AC joint reconstruction. The recommended time to RTS ranged from 12 to 48 weeks. This could be due to the term return to sports representing different degree of return to play by each program. For instance, the protocols that recommended return to play within an earlier time frame, such as 12 weeks postsurgery, may be referring to RTS with restrictions, whereas the protocols that recommended return to play after 48 weeks postsurgery may be referring to full RTS. Most protocols included in this study did not specify the expected level of return to play.

This study demonstrates that only a small percentage of academic orthopedic surgery programs (11 out of 183) published an online AC joint reconstruction rehabilitation protocol. This is similar to the findings of Makhni et al., which demonstrated that a low proportion of academic orthopedic programs published their anterior cruciate ligament (ACL) physical therapy protocols online. In this study, 33 out of 155 academic programs evaluated had ACL rehabilitation protocols published online. ${ }^{9}$ Furthermore, similar to our study, this ACL rehabilitation study also found great variation in rehabilitation components included in the different protocols. For example, only $19 \%$ of protocols made an RTS recommendation. ${ }^{9}$ Another study, performed by Trofa et al., ${ }^{7}$ found only 20 academic programs out of 155 that published online rehabilitation protocols for isolated meniscal repairs.

Another important aspect to consider regarding rehabilitation protocols is their readability. The readability measuring tool was unable to assess the readability of the protocols used in this study. Broadly, there was variation in the language used in protocols to describe rehabilitation exercises. Some protocols seemed to be geared toward patients, whereas others were intended for physicians, nurses, and physical therapists. One aspect of publishing rehabilitation protocols online is to make them easily accessible for patients. This is because patients may elect to do much of their therapy on their own at home. Home-based postoperative rehabilitation is becoming more common because of shifts in health care policy. ${ }^{9}$ Research is also indicating that some patient-driven rehabilitation has similar outcomes to more directed therapy. A study by Grant et al. ${ }^{9}$ demonstrated that recreational athletes undergoing nonacute ACL reconstruction can achieve acceptable knee ROM in 3 months following a minimally supervised rehabilitation program compared with a standard physical therapy-based program. A metaanalysis that looked at the effects of home-based rehabilitation for patients with hip fractures found home-based rehabilitation to lead to significantly improved mobility and daily activity. ${ }^{10}$ Protocols with medical terminology and abbreviations that are difficult for patients to follow can create a challenge for patients using these protocols to perform at home rehabilitation. Therefore, care should be taken to ensure that the readability of the instructions within protocols is appropriate for the targeted audience. In fact, making protocols available online will not only make it easy for patients and physical therapists to access the protocols but also create an opportunity for more comprehensive

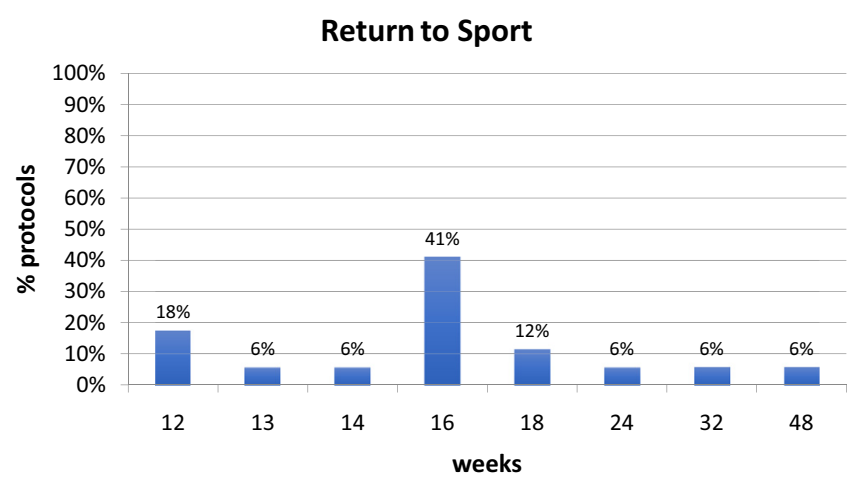

Fig 8. Recommendation by protocols for time to return to sport (in weeks) following acromioclavicular joint reconstruction. 
comparative research studies of rehabilitation protocols used by various orthopedic practices.

In addition, the protocols were variable in the amount of detail they provided to describe different rehabilitation components. Some protocols gave very specific instructions for the recommended exercises by including the time frame, the degree of movements, and the equipment needed to perform the exercises. Other protocols simply stated the name and duration of the exercises. This variation could be a result of the different audience that these protocols could be created for (i.e., patients vs physical therapists). Regardless, the protocols with nonspecific instructions are easily open to the interpretation of the reader. This can create discrepancy in the type of rehabilitation patients receive. Ultimately, this leads to nonstandardized rehabilitation practices, which can potentially result in differing postsurgery outcomes among patients.

Currently, there is no evidence-based guideline for rehabilitation following $\mathrm{AC}$ joint reconstruction. It is imperative that evidence-based practices for AC joint reconstruction rehabilitation are developed, so physicians can make more informed decisions regarding rehabilitation for their patients.

\section{Limitations}

There are several limitations to this study. First, only 21 rehabilitation protocols for AC joint reconstruction were identified on websites for academic orthopedic surgery programs and private practices in the United States. It is possible that the variability between the protocols would be less pronounced if more protocols were available. In addition, AC joint reconstruction rehabilitation protocols for academic orthopedic programs were identified by using the name of the program. However, protocols on websites for orthopedic private practice groups were identified using only a Google search. Therefore, it is possible that other private practice groups in the United States have AC joint reconstruction rehabilitation protocols available online. More protocols may have been identified if a variety of browsers had been used for the search. Finally, it is possible that many academic and private practice orthopedic surgery groups have AC joint reconstruction rehabilitation protocols; however, since those protocols were not published online, they could not be included in the study.

\section{Conclusions}

There was substantial variability in publicly accessible AC joint rehabilitation protocols, including a wide range in the recommendations for appropriate time to return to sport. Although strengthening exercises, AROM, and AAROM were recommended by most protocols, there were considerable differences in recommendations for when to initiate these rehabilitation components.

\section{References}

1. Simovitch R, Sanders B, Ozbaydar M, Lavery K, Warner J. Acromioclavicular joint injuries: Diagnosis and management. J Am Acad Orthop Surg 2009;17:207-219.

2. Kaplan LD, Flanigan DC, Norwig J, Jost P, Bradley J. Prevalence and variance of shoulder injuries in elite collegiate football players. Am J Sports Med 2005;33: 1142-1146.

3. Frank R, Cotter E, Leroux T, Romeo A. Acromioclavicular joint injuries: Evidence-based treatment. J Am Acad Orthop Surg 2019;27:e775-e788.

4. Lee S, Bedi B. Shoulder acromioclavicular joint reconstruction options and outcomes. Curr Rev Musculoskelet Med 2016;9:368-377.

5. Cote M, Wojicik K, Gomlinski G, Mazzocca A. Rehabilitation of Acromioclavicular joint separations: Operative and nonoperative considerations. Clin Sports Med 2010;29: 213-228.

6. Gowd A, Liu J, Cabarcas B, Cvetanocich G, Garcia G, Manderle B, Verma N. Current concepts in the operative management of acromioclavicular dislocations. Am J Sports Med 2019;47:2745-2758.

7. Trofa DP, Parisien RL, Noticewala MS, et al. Quality and variability of online physical therapy protocols for isolated meniscal repairs. J Knee Surg 2019;32:544-549.

8. Makhni E, Crump E, Steinhaus M. Quality and variability of online available physical therapy protocols from academic orthopaedic surgery programs for anterior cruciate ligament reconstruction. Arthroscopy 2016;32:1612-1621.

9. Grant JA, Mohtadi NG, Maitland ME, Zernicke RF. Comparison of home versus physical therapy-supervised rehabilitation programs after anterior cruciate ligament reconstruction: A randomized clinical trial. Am J Sports Med 2005;33:1288-1297.

10. Wu D, Zhu X, Zhang S. Effect of home-based rehabilitation for hip fracture: A meta-analysis of randomized controlled trials. J Rehabil Med 2018;50:481-486. 


\section{List of Protocols included in the study}

Academic Orthopaedic Programs

Loma Linda University

Massachusetts General Hospital

NYU Langone Medical Center

Saint Louis University

UConn Health

University of Colorado

University of New Mexico School of Medicine

Vanderbilt Sports Medicine

Wake Forest University School of Medicine

William Beaumont Army Medical Center

Private Orthopaedic Practice Groups

Dr. Brian Dierckman Nashville, TN

Illinois Bone and Joint Institute-Dr. Roger N. Chams

Ellis $\&$ Badenhausen Orthopaedics

Northwestern Medical Center

Orthopaedic Specialists of North Carolina

RevereHealth Orthopedics

Rockford Orthopedic

South County Orthopedics

Sports Medicine Lexington

The Stone Clinic

Twin Cities Orthopedics 